\title{
Puberty and disease activity in JIA
}

\author{
Philomine Van Pelt ${ }^{1} 2^{*}$, Anita Hokken-Koelega ${ }^{3}$, Radboud Dolhain ${ }^{4}$, Hans Bijlsma $^{5}$, Aike Kruize ${ }^{5}$, Nico Wulffraat ${ }^{1}$ \\ From 21st European Pediatric Rheumatology (PReS) Congress \\ Belgrade, Serbia. 17-21 September 2014
}

\section{Introduction}

Delayed puberty and decreased final length is reported in chronic diseases like Crohn's disease and JIA with a disease onset at prepubertal age. This may be due to systemic effects of inflammation, undernutrition or medication, for example glucocorticoids or MTX. Treatment with anti TNF has shown to restore delayed growth in JIA.

\section{Objectives}

To describe growth, onset and progression of puberty in established JIA patients who are treated intensively.

\section{Methods}

All consecutive JIA patients aged 10-24 years were asked to participate in this observational follow-up study. Demographic and disease related items were obtained yearly as well as Tanner puberty stages: Pubic Hair Girls (PHG), Breast stage (Bre), Menarche (Men), Pubic Hair Boys (PHB), Genital Stage (Gen). Reference Values were obtained from the Dutch National Growth Study. Median age at reaching each pubertal stage was estimated by Kaplan Meier survival estimates based on the data from patients of Caucasian origin and younger than 21 years. Non parametric tests are used to determine significant differences.

\section{Results}

118 girls (67\%) and 58 boys (33\%) entered the study. Thirteen percent have systemic onset type of JIA, $24 \%$ oligo- persistent type, $54 \%$ oligo-extended and polyarticular type and $9 \%$ other subtypes of JIA.

Median disease duration is 8.5 years (IQR 7,3). Median JADAS 27 is 3,8 (IQR 6.9), active joint count is $0,0(2.0)$, DAS 28 is $2,18(1,37)$. MTX is ever or currently used in $78 \%$ of the patients, anti TNF in $15 \%$ and systemic corticosteroids in $24 \%$. Early disease onset before the age of
8 years is present in $59 \%$ of the patients. Eleven patients are of non-Caucasian origin and 19 patients are older than 21 years, and are excluded from growth and puberty analysis. Median SDS length is $-0,29$ (IQR 1,38), SDS weight $-0,27$ (1,46), SDS BMI -0,08 (1.71). PHG, Bre, PHB and Gen are delayed in all stages 2-5, more pronounced in stage 5. Median delay in PHG stage 5 is 3,4 years, Bre stage 53,4 years, Menarche 3,5 years, PHB stage 5 1.6 years and Gen stage 51.7 years. Progression of puberty is more delayed in stage 4 and 5 compared to healthy Dutch children. No significant differences are seen between users and non-users of systemic corticosteroids, MTX or anti TNF. Subtype of JIA, disease activity and age at onset of JIA did not significantly influence results.

\section{Conclusion}

Although disease activity is low due to intensive treatment, puberty is still remarkably delayed. Further investigation in clinical relevance and cause of delayed puberty is needed.

\section{Disclosure of interest}

None declared

\begin{abstract}
Authors' details
${ }^{1}$ Pediatric Pmmunology and Rheumatology, UMC Utrecht, Utrecht, Netherlands. ${ }^{2}$ Rheumatology and Pediatric Pheumatology, Erasmus MC, Rotterdam, Netherlands. ${ }^{3}$ Pediatric Endocrinology, Erasmus MC, Rotterdam, Netherlands. ${ }^{4}$ Rheumatology, Erasmus MC University, Rotterdam,

Netherlands. ${ }^{5}$ Rheumatology, UMC Utrecht, Utrecht, Netherlands.
\end{abstract}

Published: 17 September 2014

doi:10.1186/1546-0096-12-S1-P151

Cite this article as: Van Pelt et al.: Puberty and disease activity in JIA.

Pediatric Rheumatology 2014 12(Suppl 1):P151. 